\title{
Fulerenos como Sensores Fluorescentes de TeMPeRATURA E de OXIGÉNIO*
}

\author{
Mário N. Berberan e Santos ${ }^{1}$ e Carlos Baleizão $0^{2}$
}

\begin{abstract}
$\mathrm{O}^{2}$ fulerenos, em especial o $\mathrm{C}_{70}$ e derivados, possuem um tipo raro e invulgarmente intenso de fluorescência, a fluorescência retardada por activação térmica (FRAT). Descrevem-se neste texto alguns dos aspectos fundamentais da FRAT e das suas aplicações em sensores de temperatura e de oxigénio baseados em fulerenos. Quase três décadas após o seu descobrimento, estas moléculas de carbono continuam a proporcionar surpresas.
\end{abstract}

\section{INTRODUÇÃO}

A descoberta dos fulerenos, em 1985, por Kroto, Smalley e colaboradores [1], quando procuravam obter cadeias lineares de carbono por vaporização de grafite com um laser, seguida da invenção de um método para a sua produção à escala macroscópica em 1990, por Krätschmer e Huffmann [2], deu início a um novo e fértil campo de investigação, que suscitou desde logo grande interesse e expectativa na comunidade científica. Uma década depois, em 1996, Kroto, Curl e Smalley receberam o Prémio Nobel de Química pela preparação e identificação da terceira forma alotrópica do carbono, entretanto estudada exaustivamente. Seguiram-se a esta os nanotubos e o grafeno (que levou a outro Nobel, desta feita de Física, em 2010). Os fulerenos têm sido aplicados em conversão de energia solar, em materiais, em óptica e na electrónica [3]. O mais comum, de longe, é o $\mathrm{C}_{60}$, no qual se apoia quase toda a química dos fulerenos, vindo depois o $\mathrm{C}_{70}$ e o $\mathrm{C}_{76}(\mathrm{Fi}$ gura 1). Estes três fulerenos podem ser vistos, quer como grandes moléculas de carbono esferoidais, quer como nanopartículas (o seu diâmetro hidrodinâmico é de aproximadamente $1 \mathrm{~nm}$ ) com composição e forma bem definidas.

A reactividade dos fulerenos levou ao desenvolvimento de diversas re-

\footnotetext{
Por decisão dos autores, e concordância da equipa editorial do QUÍMICA, este artigo não segue o novo Acordo Ortográfico

1,2 Centro de Química-Física Molecular e Instituto de Nanociências e Nanotecnologia, Instituto Superior Técnico, 1049-001 Lisboa, Portugal

E-mail: berberan@ist.utl.pt

E-mail: carlos.baleizao@ist.utl.pt
}

acções de funcionalização, com produção de derivados que aliam às propriedades intrínsecas dos fulerenos as propriedades das moléculas e grupos funcionais adicionados [4-5]. De entre as diversas reacções utilizadas para este fim, destacam-se as reacções de Prato [6] e de Bingel [7]. É interessante referir que a síntese química dos fulerenos em condições suaves não foi conseguida até hoje, sendo estes obtidos comercialmente, quer pelo método de Krätschmer e Huffmann (aperfeiçoado por Smalley), que consiste numa descarga eléctrica entre eléctrodos de grafite, em atmosfera inerte, que produz uma mistura de fulerenos (dominada pelo $\mathrm{C}_{60}$ ) e outros produtos sob a forma de fuligem, quer por reacções de combustão incompleta de hidrocarbonetos que, em condições apropriadas, dão origem a uma fuligem rica (até $20 \%$ ) em fulerenos. Em ambos os casos estes são depois separados por cromatografia. Embora os fulerenos sejam raríssimos no ambiente terrestre, descobriram-se há pouco tempo grandes quantidades dos mesmos em vários pontos da nossa galáxia, em particular numa nebulosa planetária a 6000 anos-luz da Terra (totalizando aí cerca de 1,5 massas lunares) [8].

As propriedades fotofísicas e fotoquímicas dos fulerenos [9-10] resultam do elevado número de electrões $\pi$ deslocalizados, bem como da grande simetria e da curvatura das estruturas. Note-se que, embora haja deslocalização dos electrões $\pi$ no estado fundamental, as ligações não são todas equivalentes, mantendo quase um carácter duplo e simples alternado, como se observou por difracção de raios- $X$ e, muito recentemente, de for-

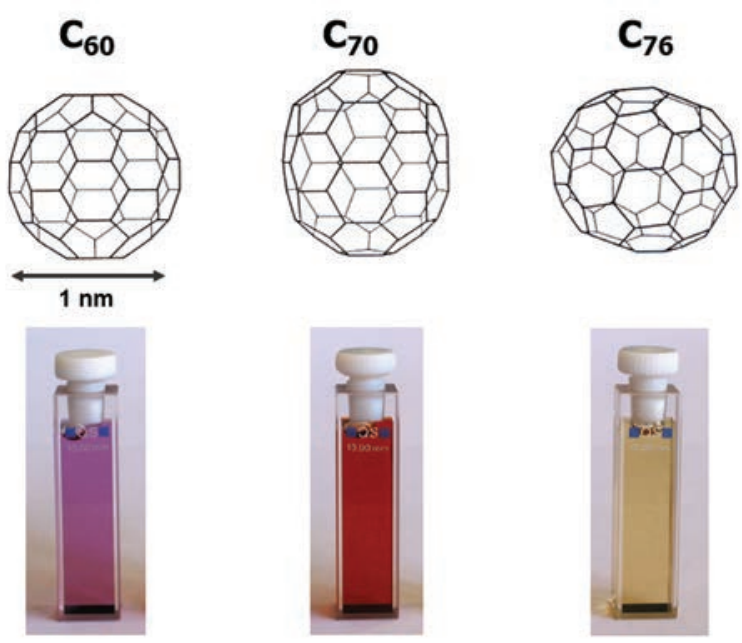

Figura 1 - Da esquerda para a direita: soluções em tolueno dos fulerenos $C_{60}, C_{70}$ e $C_{76}$. O primeiro, e mais abundante de todos os fulerenos (por razões cinéticas), produz soluções violáceas. Quanto à cor das soluções de $\mathrm{C}_{70}$, primeiramente obtidas por Roger Taylor e colaboradores em 1990, foi por este britânico designada "port-wine red". Finalmente, o C ${ }_{76}$ ' primeiro fulereno quiral, e o único não fluorescente dos três, produz soluções acastanhadas 
ma ainda mais directa, por microscopia de força atómica [11]. Os fulerenos comportam-se como polienos, e não como moléculas aromáticas, no que diz respeito à sua reactividade.

Uma das propriedades fotofísicas mais característica e interessante (para nós) dos fulerenos $\mathrm{C}_{60}, \mathrm{C}_{70} \mathrm{e}$ derivados, é a existência de um segundo mecanismo de fluorescência, a fluorescência retardada por activação térmica (FRAT, em inglês TADF, thermally activated delayed fluorescence) $[12,13]$. A FRAT é observada em muito poucas moléculas (Figura 2) e normalmente tem uma intensidade muito fraca, mas é extremamente intensa nos fulerenos, especialmente no $\mathrm{C}_{70}$.

Neste artigo iremos precisamente tratar da FRAT dos fulerenos e da sua aplicação em sensores fluorescentes de temperatura e oxigénio.
A FLUORESCÊNCIA RETARDADA NOS FULERENOS E A APLICAÇÃO DESTES COMO SENSORES FLUORESCENTES

A fluorescência molecular pode ocorrer por dois mecanismos unimoleculares diferentes: fluorescência imediata ( $F I$, ou PF, do inglês prompt fluorescence) ou fluorescência retardada por activação térmica, FRAT [12]. No mecanismo de $\mathrm{Fl}$, a emissão ocorre após absorção $S_{n} \leftarrow S_{0}$ e relaxação do estado excitado para $\mathrm{S}_{1}$. Já o mecanismo FRAT ocorre via estado tripleto. Após excitação e atingido o estado $\mathrm{S}_{1}$, ocorre um cruzamento inter-sistemas para o estado tripleto ( $T_{1}$ ou superior), seguido de um segundo cruzamento inter-sistemas de $T_{1}$ para $S_{1}$, e finalmente emissão de fluorescência (Figura 3). O ciclo $\mathrm{S}_{1} \rightarrow \mathrm{T}_{1} \rightarrow \mathrm{S}_{1}$ pode mesmo repetir-se muitas vezes antes de emissão de fluorescência, como veremos. Mas a FRAT só tem expres- são quando o rendimento quântico de formação de tripleto $\left(\Phi_{T}\right)$ e o rendimento quântico de formação de singuleto $\left(\Phi_{\mathrm{S}}\right)$ são ambos elevados [13]. Isto implica que a diferença energética entre os estados $\mathrm{S}_{1}$ e $\mathrm{T}_{1}\left(\Delta E_{\mathrm{ST}}\right)$ seja pequena e que o tempo de vida do $T_{1}$ seja longo. Para a generalidade dos fluoróforos, a FRAT é muito mais fraca que a FI e, embora já se conheça este fenómeno há muitos anos, continua a ser bastante raro [14]. A FRAT foi de facto apenas observada em alguns derivados do xanteno [15-17], cetonas [18-19] e tionas [20-21] aromáticas, numa porfirina de zinco [22], num derivado do imidazole [23], em alguns hidrocarbonetos aromáticos [24-25], em fulerenos [13, 26-30] e num complexo de cobre [31] (Figura 2).

O fulereno $\mathrm{C}_{70}$ tem um conjunto de propriedades fotofísicas quase único, e que é extremamente favorável

\section{Derivados do Xanteno}

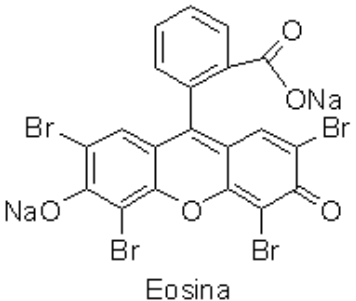

Eosina<smiles>CCOCCOc1c2c(-c3ccccc3C(=O)O)c3cc(I)c(O[N+](=O)[O-])c(I)c3oc-2c(I)c(=O)c1I</smiles>

Eritrosina<smiles>NC(=O)c1ccccc1-c1c2ccc(=O)cc-2oc2cc(O[N+](=O)[O-])ccc12</smiles>

Fluoresceína

Tionas Aromáticas<smiles>CC1(C)C(=S)c2ccccc2C1(C)C</smiles>
Porfirina de Zn

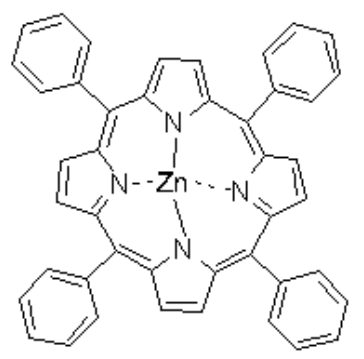

$\mathrm{C}_{60}$
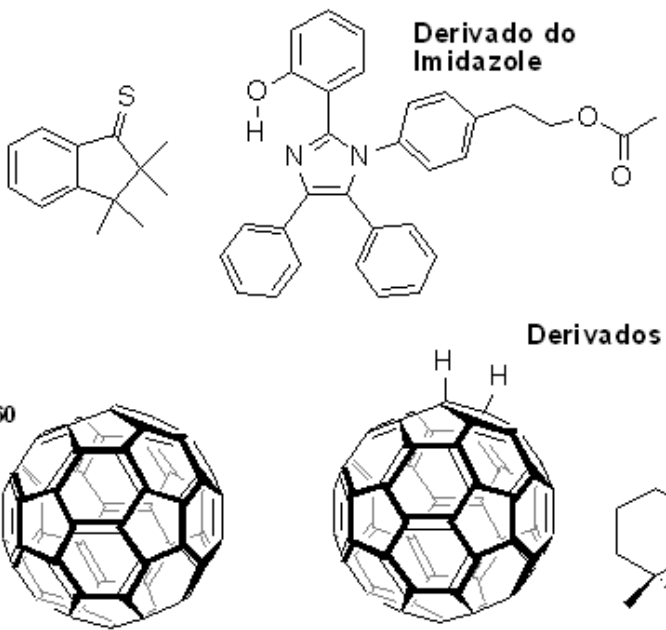

\section{Cetonas Arom áticas}<smiles>[R]c1ccc(C(=O)c2ccc([R])cc2)cc1</smiles>

$\mathrm{R}=\mathrm{R}^{\prime}=\mathrm{H}, \mathrm{F}, \mathrm{Cl}, \mathrm{CH}_{3}, \mathrm{OCH}_{3}$

Hidroc arbonetos A romáticos<smiles>CCCCCCCC</smiles><smiles>c1cc2ccc3ccc4ccc5ccc6ccc1c1c2c3c4c5c61</smiles>

Coroneno
$\mathrm{C}_{70}$

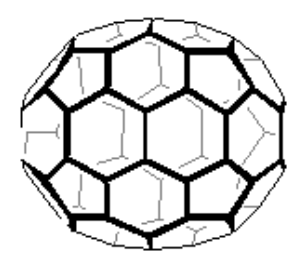

Derivados do $\mathrm{C}_{70}$
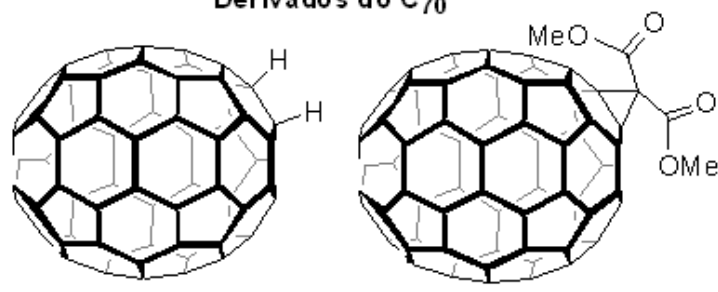

Derivados do $\mathrm{C}_{60}$
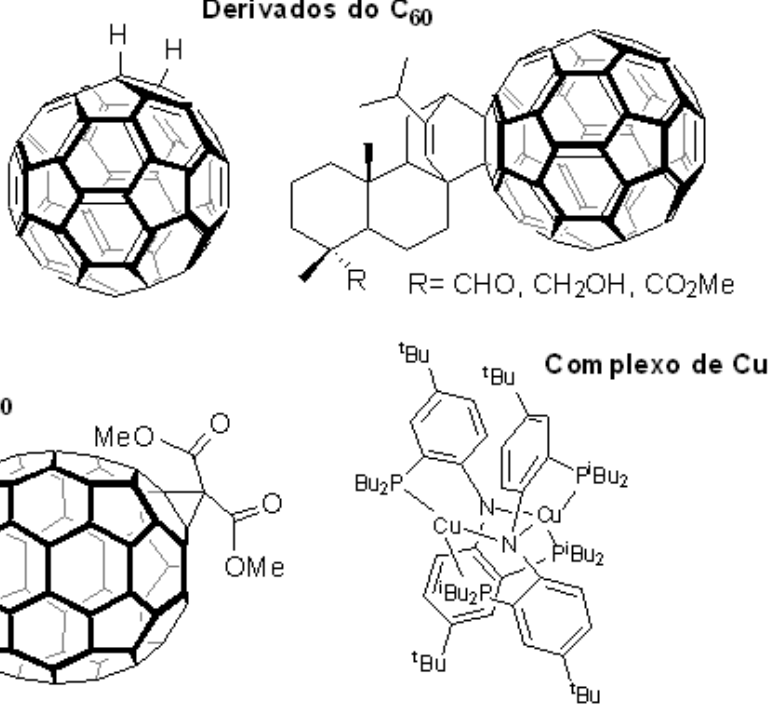

Figura 2 - Estruturas de moléculas com FRAT 
à FRAT: $\Phi_{T}$ muito próximo de 1 [32], $\Delta E_{\mathrm{ST}}$ reduzida [33], e tempo de vida de fosforescência elevado, na escala das dezenas de milissegundos [34]. Por esta razão se observa no $C_{70}$ uma FRAT invulgarmente forte [13]. Outros fulerenos, como o $\mathrm{C}_{60}$ [26] e alguns dos seus derivados [27-28], bem como derivados do $\mathrm{C}_{70}$ [29-30], também têm FRAT, mas menos intensa do que a do $\mathrm{C}_{70}$. Por exemplo, o rendimento quântico de fluorescência $\left(\Phi_{F}\right)$ global $(F R A T+F I)$ do fulereno $C_{60}$ pode ser até 10 vezes superior quando comparado com o rendimento quântico contabilizando exclusivamente a $\mathrm{FI}, \Phi_{F}^{0}$ [24], mas para o fulereno $\mathrm{C}_{70}$ (quando enriquecido no isótopo $\mathrm{C}-13$, ver adiante) este quociente pode atingir 170 [35]. Demonstra-se [30] que $\frac{\Phi_{F}}{\Phi_{F}^{0}}-1$ é precisamente igual ao número médio de ciclos $S_{1} \rightarrow T_{1} \rightarrow S_{1}$, isto é, no caso do $\mathrm{C}_{70}$ podem ocorrer, em média, até cerca de 170 ciclos completos, antes que se dê a emissão de fluorescência. Demonstra-se, ainda, que a intensificação máxima possível devida à FRAT é dada por [35] $\frac{\Phi_{F}^{\max }}{\Phi_{F}^{0}}=\frac{1}{1-\Phi_{T}}$, isto é, devido ao retor-

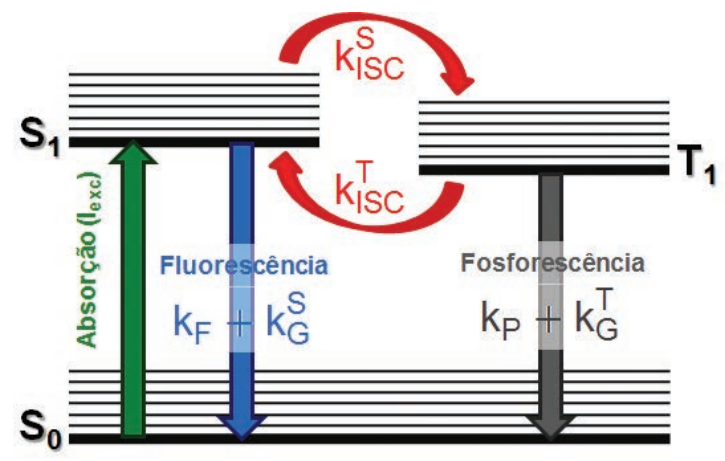

Figura 3 - Esquema cinético simplificado para a FRAT: $I_{\text {exc }}$ é a intensidade de excitação, $k_{F}$ e $k_{p}$ são as constantes cinéticas radiativas para a fluorescência e a fosforescência, $k_{G}^{S}$ e $k_{G}^{T}$ são as constantes cinéticas não radiativas para a relaxação para o estado fundamental (conversão interna a partir de $\mathrm{S}_{1}$ e cruzamento inter-sistemas para $\mathrm{T}_{1}$, respectivamente) e $k_{I S C}^{S}$ e $k_{I S C}^{T}$ são as constantes cinéticas de cruzamento inter-sistemas para a conversão singuleto-tripleto e tripleto-singuleto, respectivamente

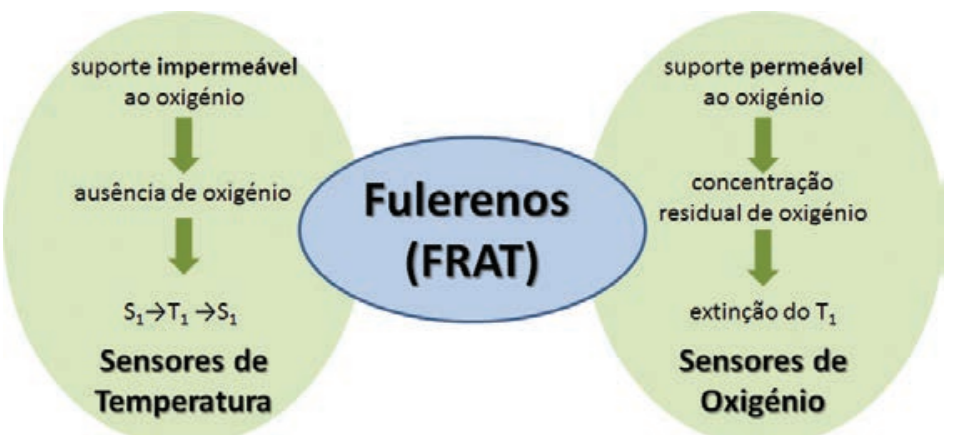

Figura 4 - Requisitos para o desenvolvimento de sensores fluorescentes com base em fulerenos vente para a molécula fluorescente, evitando-se a sua agregação (e consequentes possíveis alterações nas propriedades fotofísicas); (ii) a permeabilidade ao oxigénio terá de ser controlada. Dependendo da aplicação, será desejável uma permeabilidade elevada ou reduzida.

Os fulerenos não modificados $\left(C_{60}\right.$, $\mathrm{C}_{70}$, etc.) têm uma baixa solubilidade na maioria dos solventes orgânicos, sendo solúveis em hidrocarbonetos alifáticos e, principalmente, em hidrocarbonetos aromáticos [37]. Este comportamento limita a escolha de possíveis polímeros que podem ser compatibilizados com fulerenos. A escolha recairá em polímeros com elevada permeabilidade ao oxigénio se a aplicação for a medição de oxigénio residual, ou em polímeros com reduzida permeabilidade ao oxigénio se a aplicação for a medição de temperatura (Figura 4).

De entre os diversos tipos de sensores ópticos, os fluorescentes estão entre os mais sensíveis e versáteis [38]. Esta classe de sensores pode ser excitada e a emissão medida opticamente, não sendo necessário qualquer contacto físico, com óbvia vantagem em aplicações onde o ruído electromagnético é forte ou em que um contacto eléctrico seja indesejável. Os sensores fluorescentes têm ainda um tempo de resposta muito curto, são reversíveis, e a resolução espacial pode ir da macroescala (tintas fluorescentes) à nanoescala (microscopia de fluorescência).

\section{FULERENOS EM SENSORES FLUORES- CENTES DE TEMPERATURA}

Os sensores fluorescentes de temperatura mais comuns têm como fluoróforos complexos inorgânicos ou moléculas orgânicas [39-40], cuja intensidade de fluorescência decresce com o aumento de temperatura devido a um processo de extinção activado termicamente [41], com um limite superior de temperatura de trabalho da ordem dos $70^{\circ} \mathrm{C}$. A sua utilização para temperaturas superiores é normalmente inviável, sendo necessárias novas estratégias para ultrapassar esta lacuna. Existem ainda alguns sólidos inorgânicos luminescentes que permitem medir temperaturas elevadas, mas os 
seus tempos de vida são relativamente curtos e as sensibilidades e intensidades raramente são satisfatórias. Em [42] discutem-se alguns destes aspectos, bem como sistemas com potencial para medição da temperatura à escala dos micrómetros.

A estabilidade térmica e as propriedades fotofísicas (especialmente a FRAT) dos fulerenos sugerem que estas moléculas possam ser usadas em sensores fluorescentes de temperatura, mesmo para valores superiores a $70^{\circ} \mathrm{C}$.

A descoberta da FRAT no fulereno $\mathrm{C}_{70}$ foi o primeiro passo para o desenvolvimento de sensores fluorescentes de temperatura com base em fulerenos [13]. O estudo foi realizado numa solução de parafina líquida, e a remoção do oxigénio levou a um aumento de 20 vezes da intensidade de fluorescência, à temperatura ambiente. Ao subir a temperatura a intensidade de fluorescência também cresceu, e a $70^{\circ} \mathrm{C}$ o aumento foi de 50 vezes relativamente à temperatura ambiente na presença de oxigénio (Figura 5).
O efeito foi reversível até $70^{\circ} \mathrm{C}$, mas acima dessa temperatura a reversibilidade desapareceu (degradação da parafina). Outro problema deste sistema era a sua natureza líquida, o que limitava as aplicações.

O passo óbvio foi incorporar o fulereno $\mathrm{C}_{70}$ numa matriz sólida, aumentando desta forma a gama de temperaturas e o leque de possíveis aplicações. A chave para dispersar de forma eficiente o fulereno $\mathrm{C}_{70}$ está na escolha de um material que possa ser dissolvido no mesmo solvente que o fulereno. Existem vários polímeros nessas condições. Foram seleccionados os seguintes polímeros solúveis em tolueno (bom solvente para o fulereno $\mathrm{C}_{70}$ ): poliestireno (PS), poli(metacrilato de terc-butilo) (PtBMA) e poli(1-vinilo naftaleno) (P1VN) [43]. Os filmes foram estudados em vácuo e observou-se o mesmo efeito já descrito para a solução de parafina (Figura 6). O aumento de temperatura, até mais de $100^{\circ} \mathrm{C}$, levou a um aumento da intensidade de fluorescência, sendo o sistema reversível. Adicionou-se um padrão interno (perileno) para calibrar o sistema devi-

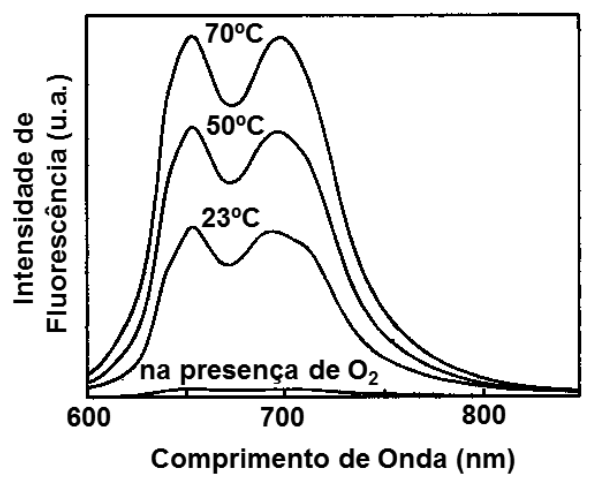

Figura 5 - Variação da intensidade de fluorescência com a temperatura para uma solução de $C_{70}$ em parafina. A emissão da solução na presença de oxigénio é independente da temperatura, enquanto que na ausência de oxigénio se observa um aumento progressivo com a temperatura. Adaptado de [13] e reproduzido com a permissão da American Chemical Society

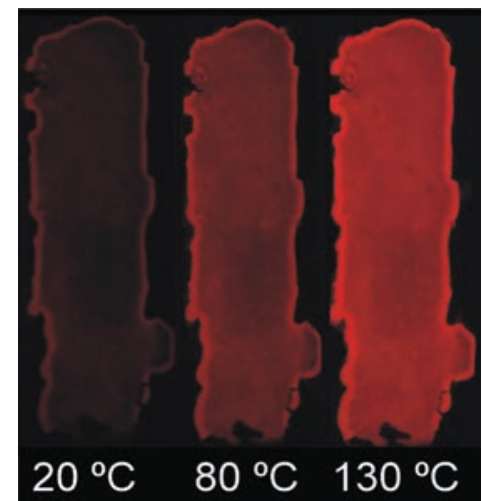

Figura 6 - Fotografias da fluorescência (visível a olho nu) do $\mathrm{C}_{70}$ num filme de PtBMA, na ausência de oxigénio. Reproduzido de [43] com a permissão da Wiley-VCH do às possíveis variações da composição do filme. O desempenho do sistema $\mathrm{C}_{70}$-PtBMA foi comparado com um sensor de fluorescência comercial, o complexo de ruténio com fenantrolina (Ru(phen) $)_{3}$ ) disperso em poliacrilonitrilo (PAN) (Figura 7). Para temperaturas acima de $70^{\circ} \mathrm{C}$, o $\mathrm{C}_{70}$-PtBMA é preferível ao $\mathrm{Ru}$ (phen) ${ }_{3}$-PAN, pois a FRAT leva a um aumento da intensidade com a temperatura. O sistema obtido é assim uma opção válida para temperaturas acima dos $70^{\circ} \mathrm{C}$, sendo o limite superior essencialmente condicionado pela resistência térmica da matriz.

A utilização do sistema anterior está no entanto limitada pelo facto de ser necessário trabalhar sob vácuo (ou em atmosfera inerte), de forma a eliminar o efeito do oxigénio sobre o estado tripleto. A incorporação do fulereno $\mathrm{C}_{70}$ num material impermeável ao oxigénio tornaria o novo sensor mais versátil, mas a fraca solubilidade dos fulerenos dificulta este procedimento. Uma possibilidade é a encapsulação em nanopartículas de polímero, que posteriormente podem ser incorporadas em outros materiais, com a possibilidade de modificar a superfície das nanopartículas para melhorar a compatibilização. Utilizou-se para este fim polimerização por miniemulsão, permitindo o encapsulamento do fulereno $\mathrm{C}_{70}$ em nanopartículas de poliestireno reticulado (com diâmetros de $60 \mathrm{~nm}$ e $190 \mathrm{~nm}$ ) [44]. As nanopartículas foram posteriormente misturadas com PAN, um polímero virtualmente impermeável ao oxigénio [45] e, após compressão e aquecimento numa prensa hidráulica, foi possível obter filmes macroscopicamente homogéneos. As propriedades de fluorescência destes filmes foram estudadas na presença e na ausência de oxigénio. Verificou-se que o tempo de vida não sofre qualquer alteração

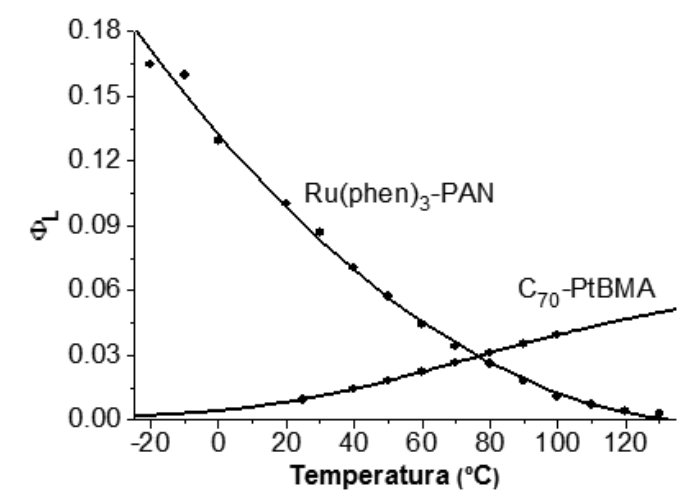

Figura 7 - Variação do rendimento quântico de luminescência $\left(\Phi_{L}\right)$ com a temperatura para $\mathrm{Ru}$ (phen) $)_{3}$-PAN e $\mathrm{C}_{70}$-PtBMA. Adaptado de [43] e reproduzido com a permissão da Wiley-VCH 
quando o filme é exposto ao ar, ou seja, a dispersão das nanopartículas em PAN impede a extinção da fluorescência retardada do $C_{70}$ pelo oxigénio. Os tempos de vida medidos são idênticos aos observados para o $\mathrm{C}_{70}$ em PS na ausência de oxigénio (Figura 8). Uma das grandes vantagens do sensor de $\mathrm{C}_{70}$ é a escala temporal dos seus tempos de vida (milissegundos), muito superiores aos do sensor comercial, $\mathrm{Ru}$ (phen) ${ }_{3}$-PAN (microssegundos), o que simplifica a aparelhagem de medição necessária. A outra vantagem, já mencionada, é a maior intensidade do sinal a temperaturas elevadas, visto que a intensidade de fluorescência aumenta com a temperatura.

\section{FULERENOS EM SENSORES FLUORESCEN- TES DE OXIGÉNIO}

A determinação de concentrações residuais de oxigénio pode ser realizada, quer por um método electroquímico, quer por um método óptico. O método electroquímico tem como base os eléctrodos de Clark [46] e é muito sensível, mas tem alguns problemas operacionais: é invasivo, é impraticável em medições simultâneas em muitos pontos de um sistema, e é de miniaturização difícil. Os sensores ópticos de oxigénio baseados nas propriedades fotofísicas de moléculas orgânicas ou complexos inorgânicos [47-48] ultrapassam as dificuldades operacionais dos sensores electroquímicos, e têm limites de detecção da ordem das parte-por-milhão em volume (ppmv). Para este fim, as moléculas orgânicas e os complexos inorgânicos são dispersos em polímeros com elevada permeabilidade ao oxigénio e com boa estabilidade fotoquímica e térmica.
A elevada sensibilidade conferida pela FRAT ao fulereno $\mathrm{C}_{70}$ levou-nos a explorar a possibilidade de utilizar o $\mathrm{C}_{70}$ como sensor fluorescente de oxigénio para concentrações residuais em fase gasosa. Para os materiais utilizados como suporte, a escolha recaiu numa sílica modificada com grupos fenilo (Ormosil, OS) e numa celulose funcionalizada com grupos etilo (EC) [49]. Ambos os polímeros são solúveis em tolueno, são estáveis termicamente e têm permeabilidades ao oxigénio muito elevadas [45]. A variação do tempo de vida de fluorescência com a concentração de oxigénio é extrema, observando-se uma redução de aproximadamente $50-60 \%$ do tempo de vida para 50 ppmv de $\mathrm{O}_{2}$ (Figura 9). Os valores de sensibilidade determinados para estes sensores variaram entre 160 e 540 partes-por-bilião em volume (ppbv), uma ordem de grandeza inferior aos valores de sensibilidade para os melhores sensores fluorescentes de oxigénio anteriormente conhecidos [47-49]. No entanto, todos os sensores fluorescentes de oxigénio têm uma resposta que depende da temperatura. Na Figura 9 vê-se que, mesmo na ausência de oxigénio, o tempo de vida diminui com o aumento de temperatura. Esta dependência pode ser tida em conta com a introdução de um sensor de temperatura. O desenvolvimento de sensores múltiplos tem crescido nos últimos anos, pois permite a partir de um ponto único obter informação sobre vários parâmetros químicos ou físicos [50]. Os sensores de oxigénio atrás descritos ( $C_{70}$ em OS e EC) foram incorporados em sensores duplos contendo um sensor de temperatura (Ru(phen) $)_{3}$-PAN) [51]. Este tem de ser insensível ao oxigénio, e após calibração dos dois

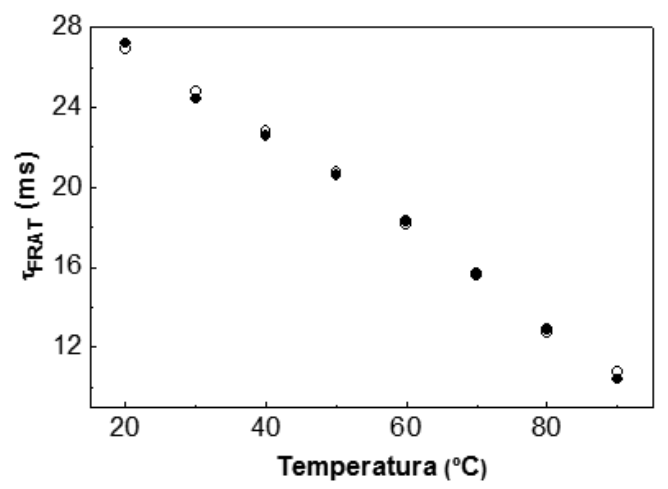

Figura 8 - Variação do tempo de vida de fluorescência $\left(\tau_{\text {FRAT }}\right)$ com a temperatura do filme PS-C 70 em vácuo (•) e do filme PS- $C_{70}$ /PAN exposto ao ar (0). Adaptado de [44] e reproduzido com a permissão da Royal Society of Chemistry sensores, é possível chegar a uma expressão empírica que permite o cálculo da concentração de oxigénio, tendo em linha de conta a temperatura de trabalho. A gama de temperaturas $\left(0-120^{\circ} \mathrm{C}\right)$ destes sensores é a mais alta até agora descrita, sendo as concentrações de oxigénio (0-0.005\%) as mais baixas da literatura.

Recentemente, descrevemos a influência da substituição isotópica $\left({ }^{12} \mathrm{C}\right.$ por $\left.{ }^{13} \mathrm{C}\right)$ nas propriedades fotofísicas do fulereno $C_{70}$, especialmente na FRAT e na fosforescência [35]. Trata-se da primeira observação de um efeito isotópico de carbono na fluorescência (e fosforescência) de uma molécula. O efeito, ainda em estudo, e que é não só de massa, mas também de spin nuclear (acoplamento hiperfino), faz com que o ${ }^{13} \mathrm{C}_{70}$ seja o mais fluorescente de todos os fulerenos (rendimento quântico de fluorescência máximo estimado de 9\%), com propriedades de FRAT bastante diferentes do seu isotopólogo ${ }^{12} \mathrm{C}_{70}$ [35]. De facto, o fulereno ${ }^{13} \mathrm{C}_{70}$ tem tempos de vida de FRAT e de fosforescência duplos dos do ${ }^{12} \mathrm{C}_{70}$, o que o torna extremamente sensível a concentrações residuais de oxigénio. A incorporação do fulereno ${ }^{13} \mathrm{C}_{70}$ em filmes poliméricos de OS, EC e PS permitiu modular a sensibilidade do sensor (na ordem dos ppbv) devido às diferentes permeabilidades dos polímeros, tornando possível a escolha do suporte de acordo com os requisitos da aplicação [52].

\section{CONCLUSÕES}

Neste artigo discutiu-se a fluorescência retardada por activação térmica (FRAT) observada nos fulerenos, bem como a sua aplicação em sensores fluorescentes de temperatura e oxigénio. Estes sensores têm desempenhos adequados para aplicações com condições de temperatura elevada $\left(>70^{\circ} \mathrm{C}\right.$ ) e baixas concentrações de oxigénio (< 1 ppmv). Não obstante o trabalho já realizado, existem ainda possibilidades de inovação. Um exemplo é o desenvolvimento de materiais mais versáteis em termos de manuseamento e processamento, com permeabilidades ao oxigénio ultra-reduzidas. Por outro lado, os sistemas descritos neste artigo têm características adequadas a uma eventual aplicação como sensores de gases raros (por exemplo krípton e xénon), devido à 


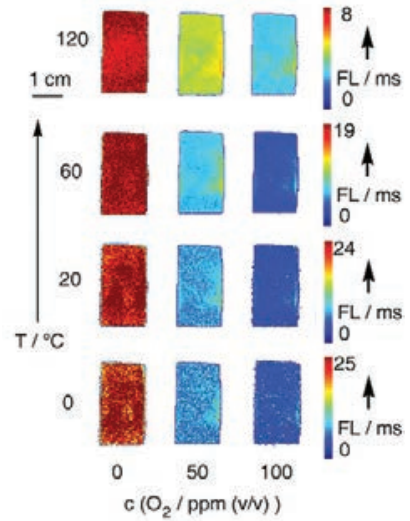

Figura 9 - Variação do tempo de vida de fluorescência (FL) com a concentração de oxigénio a diferentes temperaturas de um filme de $\mathrm{EC}-\mathrm{C}_{70}$. As imagens de tempo de vida de fluorescência estão em códigos de cores. Adaptado de [49] e reproduzido com a permissão da Wiley-VCH

sensibilidade dos fulerenos ao efeito de átomo pesado.

\section{AgRADECIMENTOS}

À Fundação para a Ciência e a Tecnologia (FCT-Portugal) pelo financiamento dos projectos de investigação PTDC/ CTM/101627/2008, PTDC/CTM-CER/ 111590/2009 e PTDC/QUI-QUI/123162/ 2010, e pelas bolsas SFRH/BPD/ 14561/2003 e SFRH/BPD/28438/2006. Ao Eng. João Garcia e à Dra. Filipa Salazar, com quem se iniciaram os estudos de FRAT, e a todos os outros socii laborum nesta linha de investigação. Ao Prof. José Paulo Farinha e à Mestre Vera Augusto (CQFM-IST) pela colaboração no desenvolvimento das nanopartículas poliméricas. Um agradecimento especial ao Prof. Otto S. Wolfbeis e ao seu grupo (Institute of Analytical Chemistry, Chemo- and Biosensors/Universidade de Regensburg/Alemanha), pela profícua colaboração ao longo dos últimos cinco anos.

\section{REFERÊNCIAS}

[1] H.W. Kroto, J.R. Heath, S.C. O'Brien, R.F. Curl, R.E. Smalley, Nature $\mathbf{3 1 8}$ (1985) 162-163.

[2] W. Krätschmer, K. Fostiropoulos, D.R. Huffman, Nature 347 (1990) 354-358.

[3] "Fullerenes: Principles and Applications", F. Langa, J.-F. Nierengarten (ed.), RSC, Cambridge, 2007.

[4] C. Thilgen, F. Diederich, Chem. Rev. 106 (2006) 5049-5135.

[5] J.L. Delgado, N. Martin, P. de la Cruz, F. Langa, Chem. Soc. Rev. 40 (2011) 5232-5241.

[6] M. Maggini, G. Scorrano, M. Prato, J. Am. Chem. Soc. 115 (1993) 9798-9799.

[7] C. Bingel, Chem. Ber. 126 (1993)
1957-1959.

[8] J. Cami, J. Bernard-Salas, E. Peeters, S. E. Malek Science 329 (2010) 11801182.

[9] D.M. Guldi, M. Prato, Acc. Chem. Res. 33 (2000) 695-703.

[10] Y.P. Sun, J.E. Riggs, Z. Guo, H.W. Rollins, 'Photoexcited state and electron transfer properties of fullerenes and related materials', in J. Shinar, Z.V. Vardeny, Z.H. Kafafi (ed.), Optical and Electronic Properties of Fullerenes and Fullerene-Based Materials, New York: Marcel Dekker (2000) 43-82.

[11] L. Gross, F. Mohn, N. Moll, B. Schuler, A. Criado, E. Guitián, D. Peña, A. Gourdon, G. Meyer, Science 337 (2012) 1326-1329.

[12] B. Valeur, M.N. Berberan-Santos, Molecular Fluorescence: Principles and Applications, $2^{\text {nd }}$ ed., Wiley-VCH, Weinheim, 2012.

[13] M.N. Berberan-Santos, J.M.M. Garcia, J. Am. Chem. Soc. 118 (1996) 93919394.

[14] C. Baleizão, M.N. Berberan-Santos, Ann. N. Y. Acad. Sci. 1130 (2008) 224234.

[15] C.A. Parker, Photoluminescence of Solutions, Elsevier, Amsterdam, 1968.

[16] S.K. Lam, D. Lo, Chem. Phys. Lett. 281 (1997) 35-43.

[17] R. Duchowicz, M.L. Ferrer, A.U. Acuña, Photochem. Photobiol. 68 (1998) 494-501.

[18] M.W. Wolf, K.D. Legg, R.E. Brown, L.A. Singer, J.H. Parks, J. Am. Chem. Soc. 97 (1975) 4490-4497.

[19] A.M. Turek, G. Krishnammorthy, K. Phipps, J. Saltiel, J. Phys. Chem. A 106 (2002), 6044-6052.

[20] A. Maciejewski, M. Szymanski, R.P. Steer, J. Phys. Chem. 90 (1986) 6314-6318.

[21] H. Eisenberger, B. Nickel, J. Chem. Soc., Faraday Trans. 92 (1996), 733740.

[22] S. Yusa, M. Kamachi, Y. Morishima, Photochem. Photobiol. 67 (1986) 519525.

[23] S. Park, O.H. Kwon, Y.S. Lee, D.J. Jang, S.Y. Park, J. Phys. Chem. A 111 (2007) 9649-9653.

[24] J.L. Kropp, W.R. Dawson, J. Phys. Chem. 71 (1967) 4499-4506.

[25] B. Nickel, D. Klemp, Chem. Phys. 174 (1993) 297-318. B. Nickel, D. Klemp, Chem. Phys. 174 (1993) 319-330.

[26] F.A. Salazar, A. Fedorov, M.N. Berberan-Santos, Chem. Phys. Lett. 271 (1997) 361-366.

[27] B. Gigante, C. Santos, T. Fonseca, M.J.M. Curto, H. Luftmann, K. Bergander, M.N. Berberan-Santos, Tetrahedron 55 (1997), 6175-6182.

[28] S.M. Anthony, S.M. Bachilo, R.B. Weisman, J. Phys. Chem. A 107 (2003) 10674-10679.

[29] S.M. Bachilo, A.F. Benedetto, R.B. Weisman, J.R. Nossal, W.E. Billups, J. Phys. Chem. A 104 (2000) 1126511269.
[30] C. Baleizão, M.N. Berberan-Santos, J. Chem. Phys. 126 (2007), 204510.

[31] J.C. Deaton, S.C. Switalski, D.Y. Kondakov, R.H. Young, T.D. Pawlik, D.J. Giesen, S.B. Harkins, A.J.M. Miller, S.F. Mickenberg, J.C. Peters, J. Am. Chem. Soc. 132 (2010) 94999508.

[32] J.W. Arbogast, C.S. Foote, J. Am Chem. Soc. 113 (1991) 8886-8889.

[33] S.M. Argentine, K.T. Kotz, A.H. Francis, J. Am. Chem. Soc. 117 (1995) 11762-11767.

[34] M.R. Wasielewski, M.P. O'Neil, K.R. Lykke, M.J. Pellin, D.M. Gruen, J. Am. Chem. Soc. 113 (1991) 2774-2776.

[35] C. Baleizão, M.N. Berberan-Santos, ChemPhysChem 12 (2011) 12471250.

[36] O.S. Wolfbeis, J. Mater. Chem. 15 (2005) 2657-2669.

[37] K.N. Semenov, N.A. Charykov, V.A. Keskinov, A.K. Piartman, A.A. Blokhin, A.A.J. Kopyrin, Chem. Eng. Data 55 (2010) 13-36.

[38] "Optical Sensors for Industrial, Environmental and Clinical Applications", R. Narayanaswamy, O.S. Wolfbeis (eds), Springer, Berlin, 2004.

[39] J.F. Lou, T.M. Finegan, P. Mohsen, T.A. Hatton, P.E. Laibinis, Rev. Anal. Chem. 18 (1999) 235-284.

[40] S. Uchiyama, A.P. de Silva, K.J. Iwau, J. Chem. Educ. 83 (2006) 720-727.

[41] J.N. Demas, B.A. DeGraff, Coord. Chem. Rev. 211 (2001) 317-351.

[42] C.D.S. Brites, P.P. Lima, N.J.O. Silva, A. Millán, V.S. Amaral, F. Palacio, L.D. Carlos, Nanoscale 4 (2012) 47994829.

[43] C. Baleizão, S. Nagl, S.M. Borisov, M. Shaeferling, O.S. Wolfbeis, M.N. Berberan-Santos, Chem. Eur. J. 13 (2007) 3643-3651.

[44] V. Augusto, C. Baleizão, M.N. Berberan-Santos, J.P.S. Farinha, J. Mater. Chem. 20 (2010) 1192-1197.

[45] J. Brandrup, E.H. Immergut, E.A. Grulke, "Polymer Handbook", Wiley-VCH Verlag GmbH, New York, 1999.

[46] R. Ramamoothy, P.K. Dutta, S.A. Akbar, J. Mater. Sci. 38 (2003) 42714282.

[47] X. Wang, H. Chen, Y. Zhao, Y. Chen, X. Wang, Trends Anal. Chem. 29 (2010) 319-338.

[48] X. Wang, O.S. Wolfbeis, Anal. Chem. 85 (2013) 487-508

[49] S. Nagl, C. Baleizão, S. Borisov, M. Schäferling, O.S. Wolfbeis, M.N. Berberan-Santos, Angew. Chem. Int. Ed. 46 (2007) 2317-2319.

[50] M.I.J. Stich, L.H. Fischer, O.S. Wolfbeis, Chem. Soc. Rev. 39 (2010) 3102-3114.

[51] C. Baleizão, S. Nagl, M. Schäferling, M.N. Berberan-Santos, O.S. Wolfbeis, Anal. Chem. 80 (2008) 6449-6457.

[52] S. Kochmann, C. Baleizão, M.N. Berberan-Santos, O.S. Wolfbeis, Anal. Chem. 85 (2013) 1300-1304. 